\title{
DESARROLLO Y PROMOCIÓN DEL MARCO NORMATIVO INSTITUCIONAL DE LA VIVIENDA ENTRE 1939 Y 1965 *
}

\section{De lo estatal a lo regional, el caso de Bucaramanga}

Néstor José Rueda ${ }^{l}$

\begin{abstract}
Resumen
Se hace un análisis retrospectivo de las políticas públicas de vivienda en Colombia durante el periodo 1939-1965, y la forma como ellas han incidido especialmente en Bucaramanga. Se parte del impulso de la vivienda obrera con los instrumentos de gestión que le fueron otorgados al Instituto de Crédito Territorial. El análisis articula aspectos de la circunstancia geopolítica mundial de los años treinta con las tendencias urbanísticas que surgieron como respuesta a esa coyuntura y que fueron adoptadas localme nte hasta los años 60 . Se explica cómo incidieron los instrumentos de gestión de la política pública durante el período mencionado y se expone el criterio del autor sobre los orígenes del desarrollo metropolitano.
\end{abstract}

\section{Palabras clave}

Vivienda, Instituto de Crédito Territorial, Área Metropolitana de Bucaramanga, política pública de vivienda

\begin{abstract}
This article analyses retrospectively the housing public policies in Colombia during the period of 1939 to 1965, and the way those policies have affected Bucaramanga. The workers housing drive with management tools that were given to the Territorial Credit Institute .These analysis articulates aspects of the global geopolitical circumstances of the thirties with urbanization trends that emerged in response to that situation, and adopted locally until the " $60 \mathrm{~s}$. The article explains how they affect the management tools of public policy during the period. Also it outlines the authors approach about the origins of metropolitan development.
\end{abstract}

\section{Keywords}

Housing, Territorial Credit Institute, Metropolitan Area of Bucaramanga, public housing policy

\footnotetext{
* El presente artículo de reflexión es parte de la tesis doctoral: "La formación del Área Metropolitana de Bucaramanga: el papel de la vivienda del Instituto de Crédito Territorial como elemento clave de su configuración" 1 Candidato a doctor en Régimen Jurídico, ordenación del territorio y la planificación urbana, Universidad de Valencia. Líder del Grupo de Investigaciones de la Facultad de Arquitectura Universidad Santo Tomás, Correo E: nergoz80@hotmail.com
} 


\section{La Creación del Instituto de Crédito Territorial (ICT): el reconocimiento de la nueva realidad demográfica}

La creación de la más importante institución de vivienda de promoción pública en Colombia, surge en el marco de una coyuntura que vale la pena resaltar. Si bien los años treinta venían precedidos de una etapa de recesión mundial, derivada de la crisis de 1929, la mala fortuna no cobijó a todos por igual; Kalmanovitz por ejemplo, sostiene que

"mientras la mayor parte del mundo capitalista se debatía en el estancamiento durante los años treinta, Colombia y otros países latinoamericanos vivieron procesos de intensa industrialización. El acelerado desarrollo industrial durante este periodo ha conducido a una interpretación bastante aceptada en el país y en América Latina, que señala la crisis como verdadero inicio de la industrialización. El estructuralismo, por ejemplo, lo caracterizó como un proceso de "sustitución de importaciones", forzado por la política intervencionista del Estado" (Kalmanovitz, 2003. p. 305).

Esta década coincidió con el periodo de la hegemonía liberal, iniciada en 1930 y prolongada hasta 1945. Es el punto de partida de una de las etapas más dinámicas de la economía colombiana y específicamente de la etapa de industrialización, proceso que se prolongó hasta finales de los setenta. Ocampo (1996. p. 125) lo define así:

"La recomposición de la actividad económica y la acumulación de capital provocaron una movilización de la población, cuya magnitud no tenía tampoco antecedente en la historia anterior del país. En especial, la de la población rural hacia las fronteras agropecuarias fue sustituida, como principal forma de migración interna, por la concentración de la población en los núcleos urbanos. En efecto, la proporción de la población que habita en las cabeceras municipales pasó de un $31 \%$ en 1938 a $39 \%$ en $1951,52 \%$ en $1964,59 \%$ en 1973 y $67 \%$ en 1985 . Las cuatro principales ciudades del país dominaron el proceso, pasando de concentrar el 8\% de la población en 1938 al 27\% en 1985 (...) El sector secundario (industria y construcción) elevó su participación del 17 al 21\%, creando una cuarta parte de las nuevas ocupaciones."

En la ciudad de Bucaramanga los efectos de la crisis se sintieron durante los primeros años de la década, lo que permite suponer que, internamente, el impacto fue de diferente intensidad al de las ciudades capitales.

Como ha sido regla en los procesos de urbanización en el mundo, las migraciones campo-ciudad son las protagonistas del crecimiento poblacional y los justificadores de los desarrollos de vivienda masiva. Colombia no escapa a este proceso, pero resulta válido destacar que las políticas en el marco del modelo de sustitución de importaciones anteriormente descrito, coinciden con una política compleja, con 
respecto a la sostenibilidad del campo y a la retención de su población. De la vivienda rural Ley 200 de 1939 a la vivienda urbana decreto 1579 de 1942

Es justamente en esta coyuntura que el gobierno de Eduardo Santos M. expide el Decreto presidencial 200 de 1939, que en su artículo segundo expone la política del Estado con respecto a la mejora y crédito para la vivienda campesina y establece la duración de cuarenta años del Instituto de Crédito Territorial. Una de las acciones que podría intuirse como un factor de estímulo para la retención del campesino en el campo, fue el establecido en La ley 46 de 1939, que dispuso diversas modalidades de estímulo a la construcción y mejoramiento de la vivienda rural. Entre ellas, la política de subsidios a las familias numerosas, con más de cuatro hijos menores "que vivieren con él y a sus expensas, a razón de $\$ 100$ por cada hijo en esas condiciones, hasta un máximo de $\$ 1.000$ por familia." Esta misma Ley autorizó la exención de impuestos y unas tarifas reducidas " $a$ costo" en materiales tanto nacionales como importados. Igualmente, la ley autorizó al Instituto y a los bancos de crédito hipotecario para importar materiales de construcción que no se consiguiesen en el mercado a precios razonables. Las políticas de vivienda eran "claras" pero la dinámica económica giraba en otra dirección, las migraciones fueron irreversibles y la política de vivienda dio un giro sustancial. ${ }^{2}$

La realidad fue más poderosa que los intentos por salvar una política de vivienda rural que desestimulara la desenfrenada migración; no fueron suficientes los seguros de vida ofrecidos para la vivienda rural, ni tampoco la "Liga Nacional de la Vivienda Rural”, presidida por el propio presidente de la república y cuya coordinación estaba a cargo del Instituto de Crédito Territorial. En este azaroso contexto, se expidió en 1942 el Decreto 1579 que determinó la creación de la "Sección de Vivienda Urbana" dentro del Instituto de Crédito Territorial:

"un nueva Sección, dotada de capital propio (inicialmente de cuatro millones de pesos. Art. $2^{\circ}$ ) y a cuyo cargo corría el suministro de préstamos para las viviendas populares urbanas, de conformidad con el decreto 380 de 1942 y con las reglas del presente decreto. Art. $1^{\circ}$ ".

Desde la perspectiva del crédito, la entidad obraría de forma similar a las facultades otorgadas al Banco Central Hipotecario a través de la Ley 170 de 1936, en la que el Instituto tenía la potestad de suscribir acciones en una compañía subsidiaria para adquirir terrenos y construir viviendas. La nueva Sección del ICT podía hacer préstamos a los municipios de acuerdo con las normas establecidas en el Decreto 380 de 1942. Era en regla, el surgimiento de la política de construcción de barrios

2 "La nueva política agraria re-aceleró la migración de campesinos a las ciudades, donde no habría trabajo ni lugar adecuado para todos. Según la Misión Currie, durante 1938 y 1945 migraron 350.000 personas a la ciudad, cifra correspondiente aproximadamente al 20\% de la población rural; entre 1938-1951 la tasa de crecimiento de la población urbana fue de $4,5 \%$ en promedio anual ( $68 \%$ por migración, $32 \%$ por crecimiento natural), mientras que en el campo fue de sólo 1.4\%." (Bejarano citado por Niño Murcia, 1980 p. 109) 
modelos, destinados a empleados y obreros, asunto claramente expuesto en los Artículos 7, 8 y 11.

\section{El higienismo como doctrina en la vivienda colombiana}

Como se demuestra en innumerables publicaciones, el higienismo surge de la preocupación constante por las condiciones insalubres en las que se desarrollaba la ciudad industrial y particularmente las viviendas a lo largo del siglo XIX. Así, los impactos sobre la salud relacionados con las construcciones, los problemas de hacinamiento, las insuficiencias de asoleamientos, servicios públicos básicos, como agua potable, aguas servidas y la disposición de los residuos sólidos, entre otras, que se relacionaban casi siempre con epidemias y pandemias, fueron motivo de debate, especialmente de las autoridades encargadas de la sanidad. ${ }^{3}$

El higienismo en Colombia se inauguró con la Ley 46 de 1918, en la que se dictaron las disposiciones para la construcción de vivienda higiénica para el sector proletario y, esta Ley coincidía con otras dictadas en Latinoamérica. A partir de allí, es reconocible el concepto de "viviendas higiénicas" destinadas a la "clase proletaria". Como era natural, la incipiente industria se concentraba en núcleos urbanos más grandes; la disposición hablaba de poblaciones de más de 15.000, que en realidad se reducía a algunas ciudades capitales. Las autoridades encargadas de la higiene debían, según la Ley, dar el aval a través del lleno de unos requisitos para su habitabilidad. Algunos otros asuntos estaban relacionados con la adquisición, las formas de pago y las tasas impositivas. A partir de esta ley la labor estatal concentra su accionar en los municipios y en algunas cooperativas creadas para el efecto.

La ley 61 de 1936 reformó el artículo 7 de la ley 46 que, en el Artículo 1, establecía los aportes obligatorios para la vivienda obrera, de acuerdo a los presupuestos anuales. En el mismo se planteó, "De preferencia se procederá a la construcción de casas colectivas en lugares centrales." De ello se puede inferir, en principio, la localización de los proyectos según las dificultades de acceso y la carencia de infraestructura y transporte adecuados en casi todas las ciudades. La mayoría de las industrias de comienzos de siglo, se ubicaba en lo que podría determinarse como las primeras periferias urbanas, incluso, dentro de lo que en la Colonia fueron los ejidos. La definición conceptual entre obrero y empleado se asumió por la Ley como equivalente $\mathrm{y}$, por tanto, fue posible que se incluyera más por el carácter uniforme en

3 "El higienismo nace con el conocido Informe Chadwick de 1839 sobre las condiciones sanitarias de la población laboral de Gran Bretaña, que tanto usarían después los padres del socialismo. A partir de esa denuncia, todas las propuestas utópicas urbanísticas tiene tintes higienistas, desde la Ciudad Jardín de Howard hasta la ciudad radiante de Le Corbusier. Zoido, F., De la Vega Sofía, Morales Guillermo, Mas Rafael, Lois, C., Rubén. Diccionario de Geografía urbana, urbanismo y ordenación del territorio. Edit. Ariel, Barcelona, 2000. 
términos del ingreso, que por una diferenciación de orden conceptual, o simplemente, en virtud de lo escaso del primero y la abundancia del segundo. Otro asunto que quedó en el vacío fue el relacionado con el concepto de "barrios obreros" y al tiempo el de "casas colectivas", que no es claro en la Ley.

En el decreto 306 de 1940 se evidenció la preocupación higienista, al determinar la realización por parte del Ministerio de Trabajo, Higiene y Previsión Social, de encuestas sociales especiales sobre las condiciones de vida y el hábitat en diferentes áreas rurales del país. Las mismas incluían variables como: viviendas rurales que existen en la región, cómo están construidas estas viviendas (disposición de las habitaciones y altura de las mismas, materiales que se han empleado en la construcción de Muros, pisos y techos), costo de estas viviendas, número de personas que habitan cada vivienda, área de la tierra que posee cada familia, cultivo que realizan estos campesinos, capacidad económica, vías de comunicación y distancia aproximada a la población más cercana, titulación de las tierras de cada familia y materiales de construcción que pueden conseguirse en la región. ${ }^{4}$

\subsection{Los Barrios Populares Modelo o Barrios Obreros: el primer ejercicio de la vivienda higienista urbana del ICT}

El decreto 1579 de 1942 que había determinado la creación de la "Sección de Vivienda Urbana" establece con claridad los principios higienistas de los proyectos

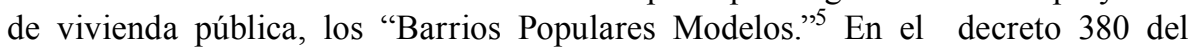
mismo año, se dictaron los principios urbanísticos, de accesibilidad, las condiciones mínimas de infraestructuras de servicios públicos, rotacionales, se describieron las condiciones de estos barrios y de sus servicios complementarios así:

“Artículo 8. Sólo se podrán construir barrios populares bajo los beneficios del presente decreto en las siguientes condiciones:

a. En lugares fácilmente accesibles, colocados dentro del área urbanizable, y con medios de transporte y comunicación adecuados;

b. con servicios de alcantarillado, acueducto y energía eléctrica;

c. observar, en cuanto a la urbanización del terreno, condiciones higiénicas y planos de las viviendas y servicios, la reglamentación que dicte el gobierno en desarrollo de este decreto".

4 Algunos elementos de este mismo formato fueron utilizados para el análisis de la situación de la vivienda obrera en Bucaramanga, desarrollado por la Contraloría General de la República. Estudios sobre las Condiciones Sociales-Económicas y el Costo de Vida de las Clases Obreras en Bucaramanga. Anales de Economía y Estadística. Revista mensual de la Contraloría General de la República, Bogotá, sep. 1946.

5 El ingeniero urbanista Rinhard Baumaister, fue uno de los precursores y defensores de este modelo en Alemania En: LUQUE, Valdivia José, Constructores de La Ciudad Contemporánea. Aproximación disciplinar a través de los textos. Departamento de Urbanismo de la Escuela Técnica Superior de Urbanismo de la Universidad de Navarra. 2004, pp. 145 - 153 
“Artículo 9. Los municipios deberán dotar a los habitantes de los barrios populares modelos de los servicios que a continuación se expresan, siendo entendido que la prestación de ellos se determinará en los respectivos contratos de empréstitos, habida consideración del número de viviendas que vaya a construirse en cada barrio y de la ubicación del mismo:

a. capilla para el culto católico, si la distancia entre el barrio y la iglesia más próxima justificare la construcción.

b. Escuelas primarias en número suficiente para el personal de niños en edad escolar que corresponda normalmente a las viviendas que haya de construirse, y restaurantes escolares;

c. sala-cuna, "gota de leche" y jardín infantil;

d. visitadoras sociales;

e. campos de deporte y recreación;

f. centro cultural y restaurante obrero;

g. plaza de mercado, si ello fuere necesario por la ubicación del barrio y número de viviendas;

h. inspección de policía, si fuere también necesaria, en consideración a las circunstancias a que se refiere el ordinal anterior."

Si bien la fase higienista, de acuerdo con los cortes temporales planteados por Saldarriaga y otros investigadores que han trabajado el tema y sobre los cuales se tiene en esta investigación la consideración genérica del concepto, se relacionan de manera directa con la vivienda pública en Colombia, es fundamental hacer la siguiente observación. A nuestro juicio, el higienismo no se agota con las normas dictadas hasta 1942, la realidad y los componentes mismos de una política de vivienda urbana, reconocible institucionalmente a partir del decreto 1579, que crea la sección de vivienda urbana del ICT, evidencia la tendencia más clara sobre el higienismo. Por tanto, esa fase no se reduce a unos planteamientos de resolución de situaciones puntuales urbanas relacionadas con los inquilinatos, los códigos de policía, las medidas sanitarias de comienzos de siglo o los problemas de vivienda rural, sino que las trasciende y pone sobre el tapete, el problema más neurálgico del higienismo y en términos sustanciales, con el crecimiento poblacional, la migración en racimo y el surgimiento de la marginalidad, que desemboca de manera irremediable en las "Zonas Negras" los "tugurios" o "chabolismo" como un problema irresoluble hasta la actualidad en prácticamente todas las ciudades del país. ${ }^{6}$

6 Constancia de ellos es el Seminario Nacional sobre urbanización y marginalidad. "como consecuencia del proceso de urbanización, proceso por el cual un país se concentra en áreas urbanas, han surgido asentamientos espontáneos de viviendas en las áreas metropolitanas, en magnitud tal que supera el crecimiento de los establecimientos controlados. Se ha calculado que mientras las ciudades crecen en una tasa de 5 a 7 por ciento al año, en las últimas décadas en América Latina, este tipo de asentamientos, denominado comúnmente como "Barrios Marginales", han crecido de 10 a 15 por ciento. Por consecuencia, las poblaciones marginales de 
El propio decreto 1371 de 1953, o “Código Sanitario Nacional” plantea las condiciones que debían cumplir las casas, apartamentos y pasajes lo mismo que las urbanizaciones destinadas a vivienda. Igualmente muestra la persistencia de los "pasajes" hoy conocidos como inquilinatos, como una modalidad legalmente aceptada de construcción de vivienda, en relación a las viviendas colectivas de la Ley 61 de 1936.

\section{La vivienda masiva y las fuentes de financiación estatal}

Una vez creada la institución y dirigidos sus esfuerzos mayores al desarrollo de proyectos urbanos de vivienda masiva, las presiones de la población suscitadas por los flujos migratorios, ${ }^{7}$ exigen una estructura capaz de dar respuesta a los requerimientos, ya no sólo del parque de viviendas, sino también a los asuntos relacionados con dos aspectos fundamentales: el acceso y la financiación, y el soporte técnico y profesional que dirija el proceso.

En términos financieros el decreto 2363 de 1944 autorizó al INSCREDIAL para otorgar préstamos a asociaciones de obreros y empleados que tuvieran por objeto construir casas de habitación para afiliados cuyo ingreso no superara los \$250.oo mensuales. En la ley 29 de 1945 se extendieron las facultades del INSCREDAL y de los municipios para otorgar préstamos a personas de la clase media económica cuyo patrimonio no excediese de $\$ 25.000$.oo. pero el ICT no obrará en solitario, el Estado para hacer frente a las demandas crecientes de habitación, crea la Caja de Crédito Agrario (1931), el Banco Central Hipotecario (1939), la Caja de la Vivienda Popular de Bogotá (1942), la Caja de Vivienda Militar (1947) y el Fondo Nacional del Ahorro (1968). Adicionalmente, operarán las cooperativas, Ley 85 de 1946, y todas ellas al unísono se van a convertir, en promotoras de la vivienda pública. Incluso el propio ICT mediante el decreto 2476 de 1953 fue autorizado para otorgar préstamos hipotecarios. El decreto reglamenta por entero la disposición. ${ }^{8}$

Dos leyes son, a nuestro juicio, claves para la estructuración de la plataforma

hoy representan aproximadamente una cuarta parte de la población total de nuestras áreas metropolitanas." Urbanización y Marginalidad. Memorias del Seminario Nacional sobre urbanización y marginalidad. Asociación Colombiana de Facultades de Medicina. Bogotá 1968.

7 Un dato esclarecedor al respecto es el aportado por Rodrigo Parra S. En el que relaciona la población así: contando como rural las poblaciones menores a 5000 habitantes, los datos relacionados son: en 1938 la población rural era de $7.136 .000(82 \%)$ y la urbana de $1.566 .000(18 \%)$, en total 8.702.000. En 1951 la rural era de 8.012.000 (70\%) y la urbana de $3.577 .000(30 \%)$, de un total de 11.589 .000 . y en 1964 la rural era de $9.617 .000(55 \%)$ y la urbana de 7.868.000 (45\%) de un total de 17.485.000. Citado por Niño Murcia, Op cit. p. 242

8 Destacamos dos artículos relacionados con la extensión del crédito, que cobra especial importancia por la cobertura que alcanza la norma: "Artículo 17. El Instituto otorga préstamos a las cooperativas de habitaciones en la cuantía y con los intereses, plazos, condiciones y modalidades que determine la Junta Directiva del Instituto. Artículo 18. Autorícese a la Junta Directiva del Instituto para conceder préstamos, con destino a la construcción de viviendas, a las entidades oficiales, semioficiales, y otras en las que el Estado es accionista. Las condiciones para estos préstamos serán reglamentadas por la Junta Directiva. 
de acción del ICT, primera la ley 85 de 1946, con la que se crean las juntas departamentales de vivienda popular, con asiento en sus capitales e integradas por el gobernador, el gerente del INSCREDIAL y representantes de los sectores industriales, agrícolas y de las cámaras de comercio; y segundo, la ley 41 de 1948 que autorizó a los consejos municipales a disponer de los terrenos elegidos para resolver el problema de la vivienda popular en las ciudades. Disposición no exenta de debates permanentes, pues como se sabe, la mayoría de las tierras a que hace mención la Ley, se referían en concreto a la pérdida de un patrimonio urbano colonial, los ejidos. ${ }^{9}$

\section{La Corporación Nacional de Servicios Públicos CNSP: el periodo de la dictadura}

El decreto 2956 de 1955 dispuso la creación de la "Corporación Nacional de Servicios Públicos", en la cual quedaron incorporados el Instituto Nacional de Aprovechamiento de Aguas y Fomento Eléctrico, el Instituto Nacional de Fomento Municipal y el Instituto de Crédito Territorial, los cuales pasaron a ser departamentos de la nueva entidad, la cual podía emitir bonos para financiación de vivienda. La creación de esta corporación fue una de las últimas medidas adoptadas por la dictadura militar y tuvo corta duración. El decreto 19 de 1957, emitido por la Junta Militar de gobierno, dispuso su disolución y creó nuevamente, como entidades autónomas, los Institutos que la constituían, restituyéndoles su patrimonio inicial. La reorganización del Instituto de Crédito Territorial se ordenó mediante el decreto 1368 de ese mismo año.

\section{El Plan de Desarrollo Lleras}

En 1958 se marca un punto de inflexión en la política de planificación del Estado, al crearse el Consejo Nacional de Política Económica y Social -CONPES- mediante la Ley 19 de 1958. Ésta se convierte en la máxima autoridad nacional de planeación y actuará como organismo asesor del Gobierno en todos los aspectos relacionados con el desarrollo económico y social del país. Al respecto, consideramos importante hacer algunas reflexiones sobre el plan que va a definir la política general del Estado entre 1961 y 1969, una vez superadas las vicisitudes de la dictadura (1953-1958) y que coincide con el arranque del Frente Nacional (1958-1974). El plan presentado

\footnotetext{
9 "Recordemos que los asuntos relativos al manejo de los ejidos fueron una constante en las deliberaciones del cabildo y que adquirieron cierta agudeza en distintos momentos. En el historial de la intervención de las autoridades municipales en materia de vivienda popular, desde la Independencia destaca la modalidad de cesión de solares y lotes de los ejidos a las familias pobres" pág. 187. MOSQUERA, T. Gilma y otros. En: SALDARRIAGA, Roa Alberto y otros, ESTADO, CIUDAD Y VIVIENDA, publicación patrocinada por el Instituto Nacional de Reforma Urbana y Vivienda de Interés Social - INURBE - Ed. Puntos Suspensivos, Editores - Consultores, Santa Fe, de Bogotá D.C. 1996
} 
por el presidente Alberto Lleras Camargo el 20 de diciembre de 1961, es el primer documento de carácter central, que elabora un diagnóstico y una política estructural de la política urbana y de vivienda en Colombia.

La Sección de Vivienda del Plan de Desarrollo, 1961, va a contar con la asesoría de la Asociación Colombiana de Planificación, la Sociedad Colombiana de Arquitectos, y la Sociedad Colombiana de Ingenieros, quienes conformaban la Comisión del Consejo Nacional de Urbanismo, creada por el Decreto 0239 de 1959. El diagnóstico apunta a la detección de los problemas sustanciales del acceso a la vivienda.

"El problema de fondo radica en el hecho de que la gran mayoría de familias de medianos y de bajos ingresos no están en capacidad de adquirir una vivienda adecuada en el mercado porque existe una fuerte discrepancia entre altos costos de producción y altos precios de alquiler o de venta, por una parte, y el reducido poder de compra, por otra. En términos económicos puede decirse que existe una alta demanda nominal pero no real, al mismo tiempo que se opera una reducida oferta porque la producción de una vivienda requiere fuertes inversiones y su mercado no ofrece las garantías de rentabilidad y liquidez que caracterizan a las inversiones en la industria y el comercio." (Plan de Desarrollo, 1961)

Se enfatiza, así mismo, que los problemas del costo del suelo y los manejos inadecuados, en términos de las técnicas y las densidades, es una de las causas más importantes de la creciente imposibilidad de acceso a la vivienda.

El Plan se va a centrar en cinco aspectos básicos: a) las limitaciones de la producción; b) acceso al mercado de viviendas; c) limitaciones socioculturales; d) el fenómeno de la urbanización; y e) las condiciones habitacionales. La importancia que comporta la revisión de estos aspectos tiene que ver con las estrategias y planes que se adoptarán para la promoción de vivienda pública, especialmente la ejecutada por el ICT.

En el primer ítem, las limitaciones de producción, se identifica como uno de los problemas cruciales, "el empleo de sistemas y especificaciones de diseño y construcción artesanales" se propone la implementación de las tecnologías empleadas para la construcción de obras públicas o privadas de gran tamaño y complejidad, en las que se utilizaban sistemas modulares, estandarizados y prefabricados, ello se calculaba, iría a disminuir los costos finales de la producción. Otro aspecto que se relaciona dentro de esta misma lógica, tiene que ver con la reducción de las áreas unitarias globales a $10 \mathrm{o} 12 \mathrm{~m}^{2}$ por persona, en contraposición a los habituales $20 \mathrm{~m}^{2}$, y agrega:

"a tiempo que las áreas unitarias por función no han sido sometidas a un proceso de racionalización que permita el máximo rendimiento de las áreas construidas." 
El debate sobre la tecnificación y la consecuente pérdida del empleo, es resuelto con el argumento que su implementación abre nuevos frentes de trabajo e implica la apertura de fuentes de producción industrial de elementos prefabricados, formaletas o molduras. La argumentación en este caso, se centraba entre la producción artesanal ligada a una mano de obra no calificada y con intervención de contratistas pequeños, dirigida básicamente a la producción de vivienda unifamiliar y con medios precarios de producción, en contraposición a procesos industriales y en serie que pudiesen aminorar los costos finales.

El acceso al mercado de viviendas, su nivel de afectación se relaciona con dos aspectos: una oferta baja y una demanda real bastante reducida, el primero ligado a los altos costos de producción y a las limitaciones de capital, y el segundo, al bajo poder de compra. De entre todas las variables anotadas, se identifica como sustancial el costo del suelo, especialmente en las ciudades capitales más importantes, donde su valor podía alcanzar hasta el 30 y el $40 \%$ del costo final de la vivienda, en tanto que en poblaciones pequeñas éste llegaba escasamente al $2 \%$. Se reconoce en la especulación del suelo, una variable que va a incidir de forma notable en la localización de los proyectos de vivienda pública y específicamente los del ICT, sobre las periferias. Asunto controversial por donde se le mire porque, si bien los precios del suelo son menores, sufren de inmediato el encarecimiento de las viviendas, por la necesaria instalación y funcionamiento de servicios públicos y la obligada adecuación de infraestructuras y dotaciones. Los costos de estas infraestructuras son asumidos directamente por el comprador y, adicionalmente, por los dineros públicos y, como se verá, estas infraestructuras son aprovechadas por igual por el sector público de manera directa y por los promotores privados de forma colateral,10 y como se podrá colegir, la distancia es un factor que se debe considerar en relación con el tiempo y los costos de desplazamiento. Aunque podría decirse, que lo que pudo ser periférico, resulta finalmente central, en virtud del factor "arrastre," aunque su relación no ha sido necesariamente directa e infalible, muchas de estas siguen siendo marginales en el sentido estructural de la palabra.

Pero no sólo la plusvalía del suelo presionaba las ocupaciones periféricas, también las propias Oficinas de Planeación a través de los Códigos Urbanos y los Planes reguladores orientaban un modelo que privilegiaba la baja densidad.11 Un primer

10 "Si se examinan, por ejemplo, los planos de los centros urbanos en donde el ICT, el Banco Central Hipotecario y las Cajas de Vivienda han realizado programas, se nota fácilmente que ellos han quedado ubicados en la periferia (...). Se comprende fácilmente la probable economía que se obtiene en el precio del lote, que puede ser amortizada en un plazo hasta de veinte años, no compensa el sobre-costo que los municipios tiene que afrontar con la prolongación innecesaria de las vías y de las instalaciones de acueducto, alcantarillado, teléfono, energía y otros servicios." (Plan de Desarrollo, 1961)

11 Así, por ejemplo, en ciudades como Bogotá, Medellín, Cali, Barranquilla, Bucaramanga, Cartagena, Cúcuta y muchas otras, se exige que cada 100 varas de tierra urbanizable sólo pueden ser construidas 25 , ya que el $35 \%$ del 
aspecto podría ser interpretado como que:

\begin{abstract}
"Normalmente no basta con disponer de suelo físicamente necesario para la edificación. Es preciso que sea aceptado como tal por el poder político. En la doble intervención de aceptación y programación del suelo radica el origen del problema urbanístico. (...) La intervención pública de programación y ordenación del suelo, produce en el espacio la división social y económica a través de una serie de elementos, técnicas urbanísticas principalmente la zonificación, densidades, definiciones tipológicas (...) de alguna forma de vivienda queda condicionada, tanto en sus aspectos formales como sociales, desde el planeamiento urbanístico" (SORRIBES citado por GAJA, 1978. p. 38).
\end{abstract}

Una hipótesis podría ser, que las regulaciones obraran de acuerdo con el propio reconocimiento de las tecnologías imperantes y la escasez de recursos, asunto que fue resuelto en la década del cincuenta y buena parte del sesenta, a partir de procesos auto-constructivos en los que la participación comunitaria fue decisiva.

Otro tema que se reconoce en el Plan es el financiero, que toca de lleno el problema de privilegio de los capitales y las sinergias que se suscitan por su implementación. Así, los capitales destinados a la vivienda comportan rotaciones más lentas 20 años en promedio con rentabilidades del $12 \%$ anual, en tanto que los créditos comerciales e industriales alcanzan niveles de rotación entre 90 días y 5 años como plazo máximo, con niveles de rentabilidad mayores, sumado a la inequidad manifiesta de insolvencia de los sectores populares en contraposición a la de estos últimos. Aunque, en sentido estricto, tendría que valorarse la vivienda no sólo desde la perspectiva del valor de cambio, sino también desde el valor de uso, como lo destaca el autor precitado.

"Decir, simplemente que la vivienda es una mercancía podría inducir a error porque no se trata de una mercancía cualquiera, que se comporta en el mercado según las leyes generales (...) La vivienda es una mercancía un tanto especial: inmóvil, cara, llena de contenidos sociales, ideológicos, simbólicos (...) en donde un aspecto clave es su carestía. Dado por su largo ciclo de producción (2 años en condiciones óptimas) un largo ciclo de vida que depende de su forma constructiva, y de su calidad 100 años en promedio. Es una mercancía valiosa que supera el ciclo vital, pero además, una mercancía de primera necesidad." (SORRIBES citado por GAJA, 1978. p. 53).

Al catálogo de dificultades planteadas hasta aquí, se le sumaba la acción descoordinada por parte de los promotores oficiales y semioficiales de la vivienda. La multiplicidad de entidades: ICT, BCH, CVM, las Cajas Municipales de Vivienda

total debe cederse para "zonas verdes" y el $40 \%$ en áreas libres. Al mismo tiempo que se imponen limitaciones al rendimiento del $25 \%$ aprovechable ya que en las "Zonas de Habitación" la altura de las edificaciones está limitada en su mayor parte de dos pisos y en el resto a tres y cuatro pisos. 
Popular y las cooperativas, obraban con total autonomía y de forma paralela. La dispersión de capitales, la carencia de normas y especificaciones de diseño y construcción, ratas de interés, cánones y plazos de amortización y de todos aquellos aspectos de racionalización.

Limitaciones socio - culturales. El acelerado proceso de urbanización derivado de los flujos migratorios campo - ciudad, mantuvieron un ideario que privilegiaba "la casa", paradójicamente reforzado por los instrumentos de planeación. El resultado espacial es predecible, la extensión sobre el territorio de proyectos de baja densidad y el agotamiento de suelos, condujo a la colonización permanente de la periferia. El Plan apuntaba a una variación urgente del modelo, que podría decirse que inicia con "Ciudad Techo o Ciudad Kennedy" en Bogotá, en 1961 y aplicada en Bucaramanga con el proyecto de Puerta del Sol en la misma década.

Dentro del fenómeno urbanizador se identifican como críticas las condiciones de marginalidad en las que se da el proceso urbano y se destacan las condiciones de precariedad y exclusión. Corrobora la tesis de que los crecimientos subnormales fueron desbordados y se enquistaron en las periferias, en donde la ausencia de infraestructuras, dotaciones y servicios básicos siempre han sido la regla.

De todos los factores anteriores se colige que las condiciones habitacionales mantenían un déficit creciente cuantitativo que se calculaba en 272.700 unidades de vivienda para 1961, aunque reconoce que los datos intercensales 1938 y 1951 no usaron conceptos similares y, por tanto, los márgenes de error podían ser importantes. Un recurso utilizado con frecuencia fue el análisis cualitativo, ligado no sólo a la carencia de la vivienda, sino también a las condiciones habitacionales, en donde los estudios de múltiples entidades eran más frecuentes y abundantes.

Figura 1. Déficit cualitativo de la vivienda obrera en Bucaramanga 1945

\begin{tabular}{|l|r|r|r|}
\hline & \multicolumn{1}{|c|}{$\begin{array}{c}\text { Viviendas } \\
\text { ocupadas }\end{array}$} & Sin sanitarios & Sin duchas \\
\hline Cabeceras municipales & 818.533 & 184.615 & 313.010 \\
\hline Otras localidades & 1.101 .516 & 977.527 & 1.073 .319 \\
\hline Todo el país & 1.120 .049 & 1.162 .142 & 1.386 .329 \\
\hline
\end{tabular}

Fuente: Anales de Economía y Estadística. Revista mensual de la Contraloría General de la República, Bogotá, sep. 1946.

En síntesis, en el Plan se van a plantear las siguientes acciones como prioritarias para subsanar el problema de la vivienda.

a. Un sistema de canalización del ahorro disponible hacia vivienda que funcionara 
como una represa de capitales para irrigar armónica y sistemáticamente diversos frentes de actividad en este campo

b. Establecer un organismo de coordinación y asistencia técnica que orientara en materia de programación, financiamiento y construcción y planificara a escala nacional la actividad

c. Estímulos a la industria de la construcción y a la formación profesional del personal dedicado a la vivienda

d. Utilización de la política fiscal e impositiva -a base de estímulos y presionesen la programación de la vivienda de interés social

e. Coordinación entre los programas de servicios públicos, comunales y asistenciales y los de vivienda

f. Regulación del rendimiento de la tierra urbana destinada a la vivienda de interés social

g. Establecimiento de normas y especificaciones de diseño y construcción de viviendas

h. Intensificación de la propiedad horizontal en vivienda y estímulos a la

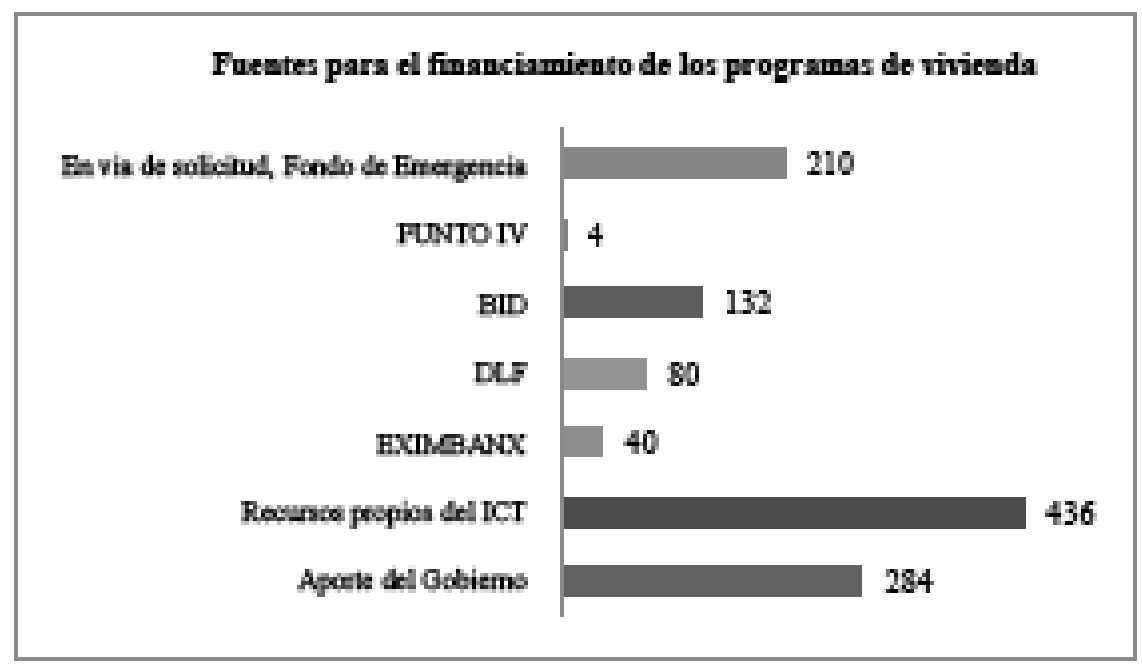

industrialización de los procesos constructivos

i. Medidas complementarias de defensa social 
j. Todas aquellas medidas necesarias para articular el sistema nacional de promoción de la vivienda de interés social.

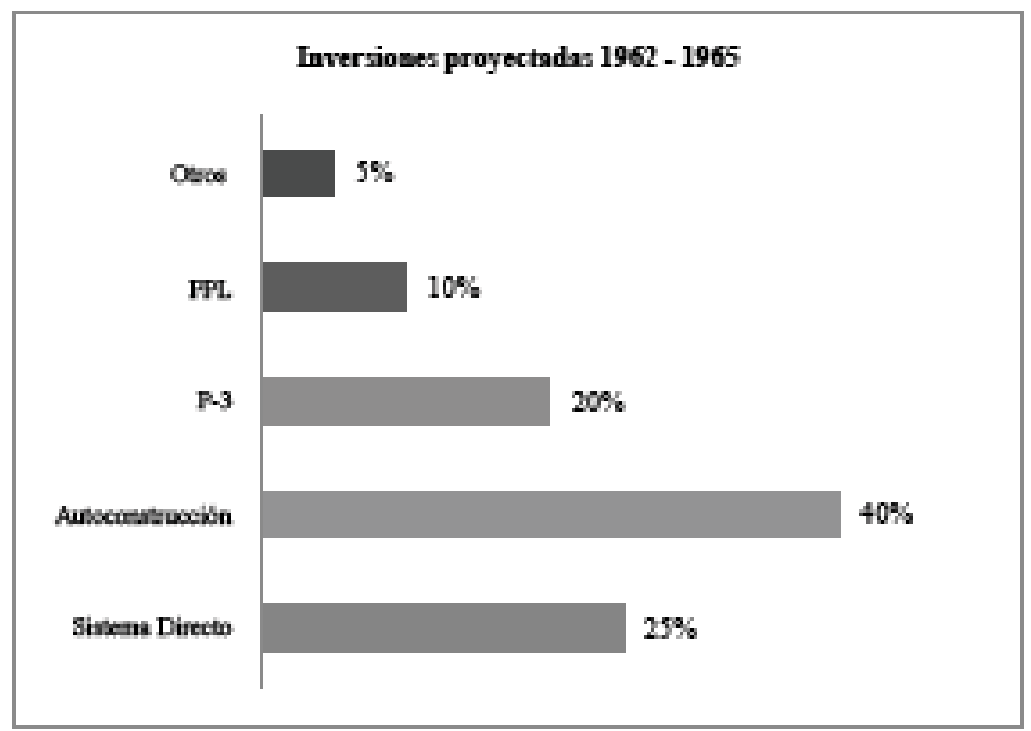

Figura 2. Fuentes de financiación de programas de vivienda

Fuente: Plan de Desarrollo 1961

Figura 3. Inversiones proyectadas por el Plan de Desarrollo

Fuente: Plan de Desarrollo 1961

De las acciones planteadas se derivan algunas reflexiones. En primer lugar, el problema de los recursos y la financiación de la vivienda contiene un sofisma bastante difundido y es aquel que muestra las "ayudas" de la Alianza Para el Progreso como un factor clave de la producción de vivienda. Si nos atenemos a los datos aportados por el Plan, para el financiamiento de la vivienda, los fondos externos fueron créditos que incrementaron la deuda pública externa. En realidad, la mayoría de los recursos provenían de la Nación, lo que hace suponer el carácter propagandístico desmedido de los agentes externos.

El desarrollo de los proyectos estuvo fuertemente ligado a la forma como se implementaron los programas, en donde la autoconstrucción fue la estrategia privilegiada para el desarrollo de los proyectos. Consistía en la venta de lotes urbanizados y un crédito de uso exclusivo para materiales de construcción, con la 
asesoría técnica del ICT. Este sistema reunió dos modalidades adoptadas en 1958 y 1959: la construcción por "ayuda mutua" en la que un grupo de familias construían sus casas bajo el cumplimiento de un mínimo de horas de trabajo obligatorio a la semana por familia, y, la construcción por "esfuerzo propio" en la que la unidad familiar edificaba la vivienda con sus propios recursos y de mano de obra. Para unificar los criterios, tanto organizativos como técnicos, el Instituto elaboró un manual de instrucciones para el manejo del proceso. ${ }^{12}$

El Sistema Directo o de Administración Directa era aquella mediante la cual el ICT acometía de manera directa la financiación, construcción y adjudicación de las viviendas. Modalidad que fue paulatinamente sustituida por sistemas de operación en los que se privilegió la acción privada en los procesos de urbanización y construcción, los Planes de Terceras Partes los P-3 que operarían de la siguiente forma: el Instituto concedía un crédito de plazos diferidos hasta seis años, con hipoteca de segundo grado e intereses del 8\% anual. En este periodo de gracia la familia debía amortizar el préstamo que le había otorgado la entidad privada bajo hipoteca del primer gado e intereses del $10 \%$ anual. ${ }^{\square}$ Un buen ejemplo de esta estrategia se puede ver en la urbanización y construcción del Barrio Lagos en casi todas sus etapas. Algo que confirma la rápida transición del modelo hacia el desmonte gradual de la acción directa del ICT y la creciente intervención del sector privado en la producción de vivienda pública. Nada despreciable fue la aplicación del sistema de Préstamos a Propietarios de Lotes, PPL, un modelo que obraría como elemento de atracción en los primeros años de la década del sesenta y que se ejecutó en forma masiva. Se dirigió específicamente a propietarios de lotes que estuvieran debidamente urbanizados y aprobados por las autoridades municipales, el Instituto elaboraba el proyecto y suministraba el préstamo necesario para la construcción de la vivienda.

\section{Conclusión}

Se puede afirmar que la acción del Estado a lo largo de estos años, se esforzó en responder a las nuevas dinámicas migratorias y la consecuente concentración urbana. Refleja así mismo, la aplicación del modelo cepalino y la fuerte injerencia de la política norteamericana en algunos países latinoamericanos, especialmente a partir de la Conferencia de Punta del Este, a través del programa de la Alianza para el Progreso. De otra parte, se constata la influencia de los modelos importados de la escuela racionalista, desarrollados por el Congreso Internacional de Arquitectura

12 En 1964 se publica la Cartilla para participantes en programas de autoconstrucción. "Consiste esencialmente en sumar los recursos y esfuerzo de la familia representados en inciativa de trabajo, mano de obra y ahorros, con el préstamo que ofrece ICT representado en lote, materiales y asistencia técnica y de servicios sociales con fines de construcción de la vivienda. 
Moderna, CIAM, fundado en 1928 y disuelto en 1959.

\section{Referencias Bibliográficas}

ACMB, Acuerdos. 16,1921; 10,1924

Estudios sobre las Condiciones Sociales-Económicas y el Costo de Vida de las Clases Obreras en Bucaramanga. Anales de Economía y Estadística. Revista mensual de la Contraloría General de la República, Bogotá, sep. 1946

KALMANOVITZ, Salomón, (2003). Economía y Nación: Una breve historia de Colombia, Escrito por Salomón Kalmanovitz, Editorial Norma, 2003

LUQUE, Valdivia José, (2004). Constructores de la Ciudad Contemporánea. Aproximación disciplinar a través de los textos. Departamento de Urbanismo de la Escuela Técnica Superior de Urbanismo de la Universidad de Navarra. pág. $145-153$

MORENO, Tanny Liliana. (2008). Historia de la salud pública en Bucaramanga. Proyecto de grado en Historia, Universidad Industrial de Santander.

MOSQUERA, T. Gilma, En: SALDARRIAGA, Roa Alberto y otros, (1996). Estado, ciudad y vivienda, publicación patrocinada por el Instituto Nacional de Reforma Urbana y Vivienda de Interés Social - INURBE - Ed. Puntos Suspensivos, Editores - Consultores, Santa Fe, de Bogotá D.C.

OCAMPO, José Antonio y Bernal Joaquín. (1996). En: Historia Económica de Colombia. Cuarta edición, Bogotá.

SORRIBES, Josep. (1984). Crecimiento urbano y especulación en Valencia. Ed., ALMUDIN de ediciones y publicaciones. Valencia 1978 p.18 citado por Gaja i Díaz, Fernando. La producción pública de la vivienda en Valencia “1939 - 1976” Tesis doctoral.

Urbanización y Marginalidad. (1968). Memorias del Seminario Nacional sobre urbanización y marginalidad. Asociación Colombiana de Facultades de Medicina. Bogotá.

VALDERRAMA, Benítez Ernesto. (1947). Real de Minas de Bucaramanga. Imprenta Departamental de Bucaramanga

Vanguardia Liberal, febrero 7 de 1925 


\section{Bibliografía Web}

CAPONI, Sandra, Entre miasmas y microbios: la vivienda popular higienizada. En: Scielo. Cad. Saúde Pública, Río de Janeiro, 18(6):1665-1674, nov-dez, 2002, formato en PDF. [Citado 2009-04-14] Disponible en: http://www.scielo.br/pdf/ csp/v18n6/13263.pdf

GONZÁLEZ ESCOBAR, Luis Fernando. Del higienismo al taylorismo: de los modelos a la realidad urbanística de Medellín, Colombia 1870 - 1932 En: Revista Bitácora Urbana Territorial, enero - diciembre, año 2007/vol. 1, número 011 Universidad Nacional de Colombia, Bogotá, Colombia, pp. 1949-1959. [Citado 2009-04-14] Disponible En: http://redalyc.uaemex.mx/redalyc/pdf/748/74811110. pdf

HIDALGO Dattwyler, Rodrigo; ERRAZURIZ Infante, Tomás y BOOTH Pinochet, Rodrigo. Las viviendas de la beneficencia católica en Santiago: instituciones constructoras y efectos urbanos (1890-1920). Historia (Santiago) [online]. 2005, vol. 38, no. 2 [citado 2009-04-13], pp. 327-366. Disponible en: $<$ http://www. scielo. $\mathrm{cl} /$ scielo.php?script $=\mathrm{sci}$ arttext\&pid $=\mathrm{S} 0717-71942005000200004 \& \operatorname{lng}=\mathrm{es} \&$ nrm $=$ iso $>$. ISSN 0717-7194

KINGMAN GARCÉS, Eduardo. Historia social y mentalidades: los higienistas, el ornato de la ciudad y las clasificaciones sociales. En: Revista de Ciencias Sociales, Diciembre 2002, No. 015 Facultad Latinoamericana de Ciencias Sociales, Quito. [Citado 2009-04-14] Disponible En: http://redalyc.uaemex.mx/ pdf/ 509/50901511.pdf

PÉREZ Luis Rubén, La Salud Pública en Santander. Historias e historiadores En: Revista Medunab, Volumen 11 De Julio 2008. Pp. 124 - 139 [Citado 2009-04-15] disponible en: http://caribdis.unab.edu.co/pls/portal/docs/PDF

Plan General de Desarrollo Económico y Social. Consejo Nacional de Política Económica y Planeación Departamento Administrativo de Planeación y Servicios Técnicos. Presentado por Alberto Lleras Camargo 20 de dic. De 1961. [Citado 2009-04-13] Disponible en: http://www.dnp.gov.co/PortalWeb/PND/ PlanesdeDesarrolloanteriores/tabid/66/Default.aspx. Formato Pdf

QUEVEDO V. Emilio. El tránsito desde la higiene hacia la salud pública en Colombia en el contexto de las interacciones internacionales. Tesis para acceder al título Phd en estudios sociales de la ciencia en la facultad de artes de la universidad Deakin, Geelong, Victoria, Australia. Octubre de 1998. [Citado 2009-04-13] Disponible en: tux.lib.deakin.edu.au/adt-VDU/uploads/approved/.../01front. pdf

RODRIGUES SOARES, Paulo R. La difusión del higienismo en Brasil y el saneamiento de Pelotas (1880 -1930) Scripta Nova. Revista Electrónica de Geografía y Ciencias Sociales. Universidad de Barcelona [ISSN 1138-9788] Nº 69 
(38), 1 de agosto de 2000 [Citado 2009-04-14] Número extraordinario dedicado al II Coloquio Internacional de Geocrítica (Actas del Coloquio) Disponible en: http://www.ub.es/geocrit/sn-104.htm

RODRÍGUEZ, Ocaña Esteban, La salud pública en España en el contexto europeo, 1890 - 1925. Ponencia publicada En: Revista San Hig Púb 1994; [Citado 200904-14] 68: 11-27 Monográfico 1994, pp. 11 - 27 disponible En: http://www. $\mathrm{msc}$. es/biblioPublic/publicaciones/recursos_propios/resp/revista_cdrom/ VOL68/68_m_011.pdf

URRUTIA, Víctor. La ideología higienista y la vivienda en Bilbao a comienzos del siglo XX. En: Cuadernos de Sección. Historia-Geografía 21. (1993) p. 239 - 344 ISBN: 84- 87471-49-8 [Citado 2009-04-14] Donostia: Eusko Ikaskuntza. Disponible En: http://hedatuz.euskomedia.org/624/ 